\title{
INHA L'T.
}

König Rudolfs Nachfolgepläne . . . . . . . . . . . . . $1-5$

Das Interregnum.

Die habsburgische Partei . . . . . . . . . . . . . . . . . 5-10

Die Oppositionspartei . . . . . . . . . . . . . . . . . . . . . $10-15$

Die Wahl von 1292.

Vorbereitungen und Parteien . . . . . . . . . . . . 15- 27

Die Wahl . . . . . . . . . . . . . . . . . . . 27-31

Albrechts Beziehungen zum Reich 1292-1288.

Belehmung, Parteien, wachsende Feindschaft . . . . . . . 31-43.

Die Verschwörung . . . . . . . . . . . . . . . . 43- 56

\section{Albrechts Nachfolge.}

Der Zug ins Reich . . . . . . . . . . . . . . . . 56- 74

Adolfs Absetzung und Albrechts erste Wahl . . . . . . . 74- 82

Die Schlacht bei Göllheim . . . . . . . . . . . . . . . 82-98

Albrechts zweite Wahl . . . . . . . . . . . . . . 98-101 



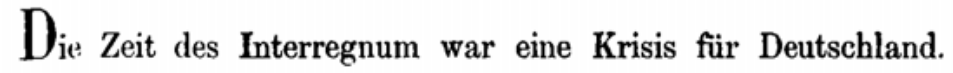
Ohnmächtiges Königthum, so lange ein solches überhaupt noch bestand, ein überaus mächtiger und zahlreicher Fürstenstand, bedeutender Einfluss von Aussen, namentlich von der Kirche her, waren Schäden, von denen der Staatskörper geheilt zu werden verlangte.

Gewaltig verändert erholte er sich aus diesem Zustande.

Aus der Menge mächtiger Grossen sonderte sich eine geringe Zahl aus, deren Macht mehr fast auf dem Herkommen als auf dem Reichthum an Land und Leuten, deren Bedeutung aber in einem bisher nicht geahnten Einfluss auf die Zustände des Reichs beruhte. Denn während früher neben der freien Wahl die Erblichkeit den Nachfolger eines deutschen Königs bestimmt hatte, schien jetzt die Erblichkeit zu Gunsten der Wahl geschwunden zu sein, und während Anfangs die Wahl Recht der ganzen Nation, dann ein Vorzug der Fürsten gewesen war, wurde sie jetzt das Privilegium eines Collegiums von Sieben. Dass diese bei der Wahl ihr Interesse ebenso im Auge haben würden, wie früher die Nation das ihre, liegt auf der Hand, und ein consequenter Schritt nur war es, wenn auch nach erfolgter Wahl die Churfürsten in Rücksicht auf ihren ferneren Vortheil das Königthum controlirten.

Diese Verhältnisse vor Allem traten in den ersten Zeiten nach dem Interregnum hervor. An sie schlossen sich dann weiter die Bemühungen, die königlich-churfürstliche Macht dem 
Papst gegenüber zu fixiren, an, welche das erste Drittel des 14. Jahrhunderts erfüllen und ihren Ausdruck und ersten Ab. schluss in dem Churverein erhalten.

Aber wider die Ansprüche der die freie Wahl vertretenden Churfürsten erhob sich Rudolf von Habsburg, indem er die Nachfolge zu bestimmen, sie seinem Geschlechte zu sichern suchte.

Diese Bemühungen Rudolfs bilden den Ausgangspunkt unserer Betrachtung.

Ein anderes Bestreben von ihm, das darin bestand, seinem Geschlechte durch Gründung einer Hausmacht grössere Stärke zu geben, ward mehr vielleicht als durch eine Berechnung von seiner Seite durch die Hand des Schicksals mit jenem Plane, die Krone bei seinem Geschlechte erblich zu machen, verknüpft.

Denn Anfangs hatte er die Nachfolge dem zweiten seiner Söhne, Hartmann, seinem Liebling zugedacht. Doch als der in den Wogen des Rheins ertrank, und als hernach auch Rudolf ! sein jüngster Sohn, dem er nach Hartmann die Nachfolge gesichert hatte, gestorben war, da wagte er es, fü Albrecht, den Aeltesten seiner Söhne, jetzt den Einzigen, um die Nachfolge zu bitten 2 . Nicht geringere väterliche Liebe war der Grund, dass er für seinen ältesten Sohn zuletzt um die Krone warb, aber mit Recht erschienen ihm die zwei grossen Arbeiten getheilt leichter vollfuhrbar, als wenn er sie ohne Noth in Eines Hände legte. Die selbstständigere und andauerndere und daher schwierigere Arbeit aber war die Gründung einer Hausmacht. Rudolf hatte wohlgewählt, da er Albrecht für sie ausersah. Denn dieser führte

' Die Nachfolge Rudolfs bewilligt Wenzel von Bohmen (Urkuade vom 13. April 1290), sobald König Rudolf die Kaiserkrone erlangt babe. Von der Kaiserkrōnung Rudolfs war aber schon seit Januar 1286 die Rede. ( $\forall$ gl. Böhmer S. 129 zum 20. Jan.) Dadurch erklärt sich auch Ellenhards Nachricht: Mon. SS. XV ,obiit Rudolfus . . . cum creari deberet auxilio patris in regem Romanorum pater vero in imperatorem. “

2 Die 1753 zu Frankfurt (in Quart) erschienene „comment. hist. de ultima Rudolfi cura, ut filius etc." von Beulwitz habe ich nicht erhalten können. 
sie unter vielen Kämpfen durch wie es in seinem Wesen lag: unverzagt, entschieden, aber leidenschaftslos.

Die Hausmacht bestand, als Rudolf seine Bemühungen begann, Albrecht die Krone zu sichern.

Den ersten Versuch machte er auf dem Reichstage zu Erfurt (13. August 1290). Albrecht erschien mit gewählter Pracht, wie er es liebte, wenn sie grösseren Vortheil zu versprechen schien. Aber die Verhandlungen führten nicht zu dem erwünschten Resultat. Freilich fehlt es an bestimmten Nachrichten. Doch schon aus dem Schweigen der Berichterstatter, das, wenn wirklich positive Resultate erzielt worden wären, mit Recht auffiele, lässt sich die Erfolglosigkeit vermuthen. Mehr beweist die noch zu Erfurt erfolgte Belehnung Albrechts mit dem durch Ladislaus Tode ledig gewordenen Königreich Ungarn 1. Es scheint, als ob Rudolf, ahnend oder schon erkennend, dass seine Bemühungen um die Nachfolge fruchtlos bleiben würden, die Thätigkeit seines Sohnes wieder auf jene Aufgabe lenkte, ihm neuen Boden zur Vergrösserung der habsburgischen Hausmacht zuwendend. Die Fürsten werden schon diesen ersten Versuch ungern gesehen haben, doch wird ein Theil derselben für Albrecht gewesen sein. Vor Allen stand der Pfalzgraf Ludwig zu ihm, wie aus dem Versprechen, welches Albrecht demselben am 9. September zu Regensburg giebt 2, ihm, „falls er König würde", alle Gerechtsame \%u bestätigen, erhellt. Es ist zugleich ein Beweis dafür, dass Albrecht trotz jenes vereitelten Versuchs die Hoffnung auf die Nachfolge im Reich nicht aufgegeben hat.

Eben so wenig Rudolf. Die böhmische Stimme, auf die er für Rudolf hatte zählen können, die also, wennschon sie zu Erfurt Albrecht entgegen gewesen war, doch nicht unbedingt dem babsburgischen Interesse zu widerstreben schien - wie denn Wenzel auch durch seine Gemahlin Gutta Rudolfs Schwiegersohn war -

${ }^{1}$ Die Urkunde vom 31. Aug. 1290; s. Böhmer Reg. No. 1070.

${ }^{2}$ Die Urkunde gedruckt in den Quellen und Frörterungen z. bayr. Gesch. V. 447. 
sie vor Allem suchte er zu gewinnen. Am 26. September belehnte er zu Erfurt Wenzel mit dem Fürstenthum Breslau und Schlesien '; an demselben Tage beurkundete er ihm den Besitz des Schenkenamts, wonach er bei der Wahl eines deutschen Königs gleich andern Wählern Wahlrecht und Stimme haben sollte.

Auf einer Versammlung zu Frankfurt brachte er die Successionsfrage von Neuem zur Sprache. Die Churfürsten waren uneinig 2. Man ging auseinander, ohne dass es zu dem von Rudolf gewünschten Resultate gekommen wäre ${ }^{3}$.

Bald nach der Frankfurter Versammlung starb der König Warum die Churfürsten seinen Vorschlag in Betreff Albrechts nicht annahmen, da sie sich doch der Nachfolge Rudolfs durchaus nicht so bestimmt widersetzt hatten, liegt auf der Hand. Albrecht war derjenige der Habsburger, der im Besitz der österreichischen Erblande war; und indem ihm auch noch Ungarn zufiel, konnte die Furcht der Fürsten vor seiner Nachfolge nur wachsen. Vielleicht auch scheuten sie seine Persönlichkeit: denn die Energie,

${ }^{1}$ Urkunde b. Böhmer Reg. No. 1075.

2 Vgl. Chron. Sampetrin. (Mencken SS. R. G. III.) und Cont. Ratisbon. (Mon. SS.XV. 416.) Die Annal. brev. Wormat. (Mon. SS. XV. 78) sagen nur "contradicentibus electoribus. Die Nachricht von Thomas Ebendorf ab Hasselbach (lebte um 1430) bei Pez SS. rer. Austr. II. 754 ist wohl zu verwerfen. Sie lautet: Interea Dux Albertus a septem Electoribus in Regem Romanorum eligendus sperificatur, quorum \& nuntios suscepit, interpellantes, ut se quantocyus ad locum electionis Francofordiam per suum ascensum disponeret; dum Gerhardus Moguntinus .... apud Rudolphum Regem monita surrogaret in regno \& de surrogatione vel Alberti Comitis de Hayerloch vel Alberti ducis Austriae ab eo sciscitans comperisset."

${ }^{3}$ H. A. H. Berduschek "Graf Albrecht von Zollern-Hohenberg und sein Verhältniss zu Rudolf I. und K. Albrecht I.“ 1853. S. 39 erwähnt, dass Rudolf, als er erkannte, dass er Albrecht die Nachfolge nicht zu sichern vermöchte, sie dem Grafen Albrecht von Heigerloch, seinem Schwager, zuzuwenden gesucht habe. Riedel theilt mit Beziehung auf ihn dieselbe Nachricht mit. Auf welche Quellen sie sich stutzt, habe ich nicht ausfindig machen können. (Nur die in der vorigen Anm. angeführte Nachricht Ebendorfs enthălt etwas derartiges.) Da von den gleichzeitigen Ueberlieferungen keine das hier Erzählte erwähnt, ist es zu verwerfen. 